ISSN-i 1390-7042 | IssN-e 2602-8484

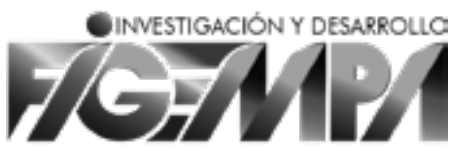

\title{
COMPORTAMIENTO DEL MARGEN DE REFINACIÓN EN EL SECTOR HIDROCARBURÍFERO DEL ECUADOR EN EL 2018
}

\section{Behavior of the refining margin in the hydrocarbon sector of Ecuador in 2018}

Carlos Carrillo Villavicencio Universidad Central del Ecuador ORCID: https://orcid.org/0000-0002-9999-0467

\author{
Rony Parra Jácome \\ Instituto de Investigaciones Hidrocarburíferas \\ Universidad Central del Ecuador \\ ORCID: https://orcid.org/0000-0003-2942-7449
}

\section{Recibido: septiembre de 2020 \\ Aprobado: mayo de 2021 ARTÍCULO ORIGINAL}

DOI: $10.29166 /$ revfig.v11i1.2630

PALABRAS Clave Refinación, margen bruto de refinación, margen neto de refinación, costo de refinación, procesos de refinación.

KEYWORDS Refining, gross refining margin, net refining margin, refining cost, refining processes.

\section{RESUMEN}

El margen de refinación es un indicador financiero que diferencia los ingresos de los productos refinados y los costos del petróleo crudo y otros insumos que intervienen en el proceso de refinación. Este indicador también puede ser mejorado, incluyendo costos adicionales por la operación y mantenimiento de la infraestructura de refinación, lo cual acerca su resultado a un margen neto de producción. La dinámica del margen de refinación está condicionada por la eficiencia técnica en términos de diseño y operación de una refinería, así como también por las condiciones de precios del petróleo y sus derivados en los mercados internacionales. El margen de refinación del sector hidrocarburífero del Ecuador fue analizado para fijar puntos de referencia sobre la rentabilidad del negocio de refinación en el país. Se identificaron los flujos de los productos finales que se obtienen en cada una de las refinerías, la materia prima (crudo Oriente), diluyentes, condensados y/o productos importados que se utilizan para el proceso del blending. Además, se reconoció los costos de operación para obtener los productos terminados bajo parámetros de calidad normados por el Estado, que luego son comercializados en el mercado interno y externo. Los precios de las materias primas y productos finales fueron obtenidos a partir de un benchmark de agencias internacionales, publicados de manera mensual e inferidos de acuerdo a la calidad del petróleo y derivados, que son utilizados y producidos en las refinerías nacionales. El estudio muestra que la dinámica del margen bruto de refinación cambia de acuerdo a la variabilidad del precio internacional del petróleo, ya que los precios de los refinados se encuentran indexados al precio de la comercialización del petróleo. Aunque este estudio no pretende informar sobre la política energética, sino mostrar una metodología transparente para analizar la rentabilidad de las diferentes refinerías aplicada al caso ecuatoriano, los resultados obtenidos pueden ser una aproximación para informar la situación de las refinerías con fines de política pública, para lo cual es preciso que los datos sean analizados y mejorados en futuros trabajos. En el 2018 las refinerías de Esmeraldas, La Libertad y Shushufindi mostraron valores promedios mensuales del margen bruto entre USD 2,3 a 9,4 dólares por barril, sin embargo, de acuerdo a los informes de EP Petroecuador el costo de operación promedio de las tres refinerías en el año de estudio fue de 6,24 dólares por barril, lo cual reduciría su competitividad en el mercado. 


\begin{abstract}
The refining margin is a financial indicator that differentiates the income from refined products and the costs of crude oil and other inputs involved in the refining process. This indicator can also be improved, including additional costs for the operation and maintenance of the refining infrastructure, which relates to its result at a net production margin. The dynamics of the refining margin is conditioned by the technical efficiency in terms of the design and operation of a refinery, as well as the price conditions of oil and its derivatives in international markets. The refining margin of the hydrocarbon sector in Ecuador was analyzed to set benchmarks on the profitability of the refining business in the country. The flows of the final products obtained in each of the refineries, the raw material (crudo Oriente), diluents, condensates and/or imported products that are used for the blending process were identified. In addition, the operating costs were recognized to obtain the finished products under quality parameters regulated by the State, which are then commercialized in the internal and external market. The prices of raw materials and final products were obtained from a reference point from international agencies, published monthly and inferred according to the quality of oil and derivatives, which are used and produced in national refineries. The study shows that the dynamics of the gross refining margin changes according to the variability of the international price of oil, since the prices of refined products are indexed to the price of oil commercialization. Although this study does not intend to report on energy policy, but rather to show a transparent methodology to analyze the profitability of the different refineries applied to the Ecuadorian case, the results obtained can be an approximation to inform the situation of the refineries with public policy fines, to which is necessary that the data be analyzed and improved in future work. In 2018, the Esmeraldas, La Libertad and Shushufindi refineries showed average monthly gross margin values between USD 2.3 to 9.4 dollars per barrel, however, according to reports by EP Petroecuador, the operating cost of the three refineries in the year of study was 6.24 dollars per barrel, which would reduce their competitiveness in the market.
\end{abstract}

\section{INTRODUCCIÓN}

El proceso de refinación corresponde a la etapa intermedia de la cadena de valor de los combustibles fósiles, que va desde la exploración de reservas petroleras, extracción de petróleo, transporte, procesos de refinación para finalmente distribuirlos y comercializarlos en el mercado local o internacional para sus usos finales en la sociedad.

El sistema ecuatoriano de refinación consta de tres refinerías principales y algunas plantas topping que sirven para el autoconsumo en los procesos de extracción de petróleo (no fueron consideradas en este estudio) i) Refinería La Libertad-RLL con capacidad de procesamiento de 45 mil barriles por día, empezó sus operaciones en 1968 y fue diseñada a partir de los procesos de destilación simple para procesar el petróleo ligero proveniente de los campos del litoral, principalmente de yacimientos del campo Ancón y otras mezclas de hidrocarburos; ii) Refinería estatal Esmeraldas-REE, empezó sus operaciones en 1977 con una capacidad de procesamiento de $98 \mathrm{mil} \mathrm{ba-}$ rriles por día, luego en 1997 se incrementó a 110 mil barriles por día, capacidad que se mantiene hasta la actualidad. Cuenta con sistemas de fraccionamiento catalítico y reformado de naftas que ayudan a optimizar los productos livianos como GLP, gasolinas y diésel; iii) Complejo industrial Shushufindi-CIs, inició sus operaciones en 1987 con una capacidad de procesamiento de 10 mil barriles por día, luego en 1997 se realizó una ampliación a 20 mil barriles por día, además cuenta con una planta de gas que procesa el gas asociado provenientes de los campos petroleros del Oriente, con una capacidad de procesamiento de 25 millones de pies cúbicos de gas por día (ver Figura 1).

El parque refinador del Ecuador cuenta en la actualidad con una capacidad nominal instalada para el procesamiento de 175 mil barriles de petróleo por día que se ha mantenido estática desde 1997, fecha en la que se amplió la capacidad de la refinería de Esmeraldas a 110 mil barriles por día (EP Petroecuador, 2018). 
Esta capacidad de procesamiento no es suficiente para abastecer la creciente demanda nacional de combustibles. En el 2018 se consumieron 91 millones de barriles de refinados, mostrando un crecimiento del 5\% con respecto al año anterior. La elevada dependencia de las sociedades a los combustibles fósiles muestra un rápido crecimiento en el consumo; por ejemplo, en el período 2000-2019 la demanda del Ecuador en gasolinas y diésel creció en promedio 5\% anual y el GLP en 3\% anual.

Como se observa en la figura 1, la capacidad de refinación estática y el rápido crecimiento del consumo de refinados, generan un desequilibrio entre la oferta y la demanda, por lo cual el Estado se ve en la necesidad de cubrir esa brecha con combustibles importados para garantizar el abastecimiento seguro de derivados y mantener la dinámica socioeconómica del país. En el mismo período de análisis las importaciones crecieron en promedio un 13\% anual, reflejando un total por importación entre gasolinas, diésel y GLP de 49 millones barriles en el 2018 (ver Figura 2).

De acuerdo con la clasificación por tipología de refinerías propuesta por El-Gendy and Speight (2015) se muestra que las refinerías de Shushufindi y La Libertad mantienen procesos de baja conversión (unidades de destilación simples), mientras que la refinería de Esmeraldas se la considera como de mediana conversión por sus procesos de mayor complejidad (unidad de fraccionamiento catalítico fluidizado, FCC), no obstante, el parque refinador no cuenta con procesos de alta y profunda conversión que involucren unidades para procesamiento de residuos y mejoramiento de los estándares ambientales (ver Figura 3). En el 2019 las refinerías produjeron producto residual en un $30 \%$ en Esmeraldas, $47 \%$ en Shushufindi y $61 \%$ en La Libertad como se muestra en la figura 3 (Petroecuador, 2019).

A su vez, el deterioro de la calidad del petróleo por la escasez de reservas de crudo liviano y medio en los campos de la Amazonía ecuatoriana puede afectar a la productividad de la infraestructura instalada de refinación, cuyo API de diseño fue de 23 a 30 para Esmeraldas, 28-30 para La Libertad y 29 para Shushufindi (Baquero, 2006).

En los últimos 12 años han existido intenciones del Estado para modernizar y ampliar la capacidad de procesamiento de las refinerías. En 2008 se creó la empresa mixta Refinería del Pacífico entre Ecuador y Venezuela para construir una refinería de alta conversión con capacidad de procesamiento de $300 \mathrm{mil}$ barriles por día (RDP plan institucional, 2016). Entre junio de 2014 y febrero del 2015 se realizaron trabajos de modernización en la refinería de Esmeraldas en cuyo caso se recuperó la capacidad de procesamiento de la FCC y otras unidades y utilidades de la refinería que se encontraban con problemas de operación (EP Petroecuador, 2015). El 10 de julio de 2020 el Estado, vía Decreto Ejecutivo N. ${ }^{\circ}$ 1094, decidió «autorizar con carácter excepcional la delegación a la iniciativa privada de la gestión conjunta de la refinería Esmeraldas con la EP Petroecuador» para generar las inversiones en procesos de alta conversión y mejoramiento de la calidad de los combustibles (Decreto Ejecutivo $\mathrm{N}^{\circ}$ 1094, 2020).

También se planteó nuevamente la necesidad de incrementar la capacidad instalada del país con la construcción de una nueva refinería que tendría una capacidad de refinación de entre 150 y 300 mil barriles de petróleo por día que incluiría plantas de conversión profunda para la producción de combustibles bajo la norma Euro 5, es decir, con bajas cantidades de azufre (Ortiz, 2020).

Es clara la preocupación y la necesidad del Estado por modernizar y mejorar la infraestructura de refinación del país, sin embargo, su estrategia ha resultado poco efectiva. La operación de las refinerías nacionales no logra abastecer la demanda nacional de derivados y parte de la producción de combustibles no consigue cumplir con los parámetros de calidad en términos de contenido de azufre y octanos que se estipula en la norma INEN 935 para gasolinas e INEN 1489 para diésel, por lo cual, dentro del modelo de gestión se realizan mezclas (blending) con productos importados para cumplir los parámetros establecidos en la normativa nacional (EP Petroecuador, 2019).

Los problemas arriba detallados, reducen la competitividad de las refinerías en el Ecuador, lo cual se ve impactado, además, por las fluctuaciones de los precios de la materia prima y de los derivados, lo cual se mostrará en el desarrollo de este estudio al analizar el parque refinador, calculando el comportamiento de los márgenes de refinación de las tres refinerías del país.

\section{METODOLOGÍA Y OBTENCIÓN DE DATOS}

El margen bruto de refinación es utilizado como un indicador de la rentabilidad de las operaciones de una refinería (Ramkumar, 2016). Se calcula relacionando los ingresos totales de las ventas de productos refinados menos el costo del petróleo crudo y otras materias primas. El margen neto se define como el margen bruto menos los costos de comercialización de productos derivados del petróleo, los costos internos de energía y otros costos operativos (Gary et al., 2004).

De acuerdo con Lluch Urpi (2011), la función económica para el margen neto está definida por la 


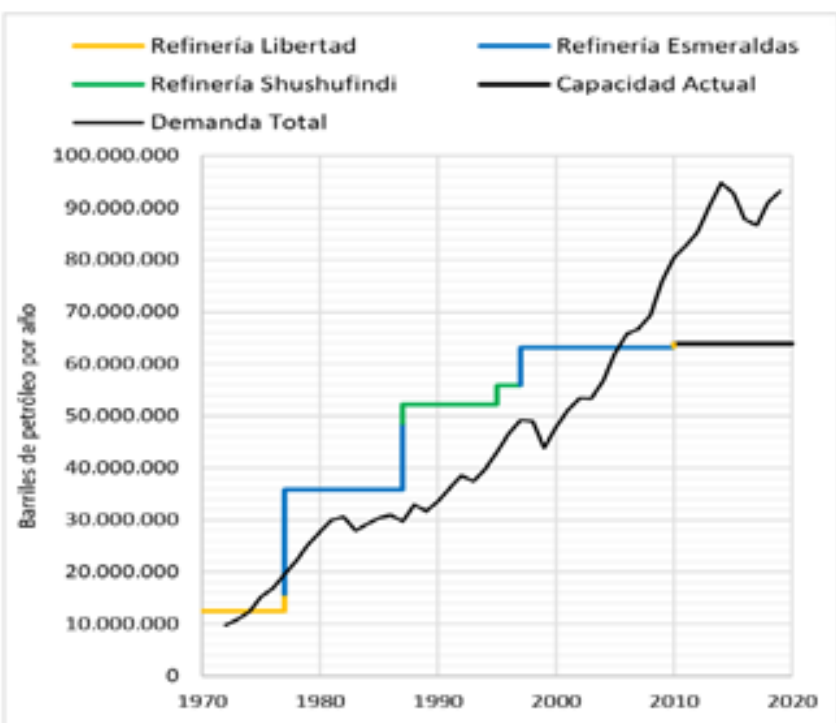

Figura 1. Construcción de las refinerías y crecimiento de la demanda nacional de derivados. Fuente: EP Petroecuador.

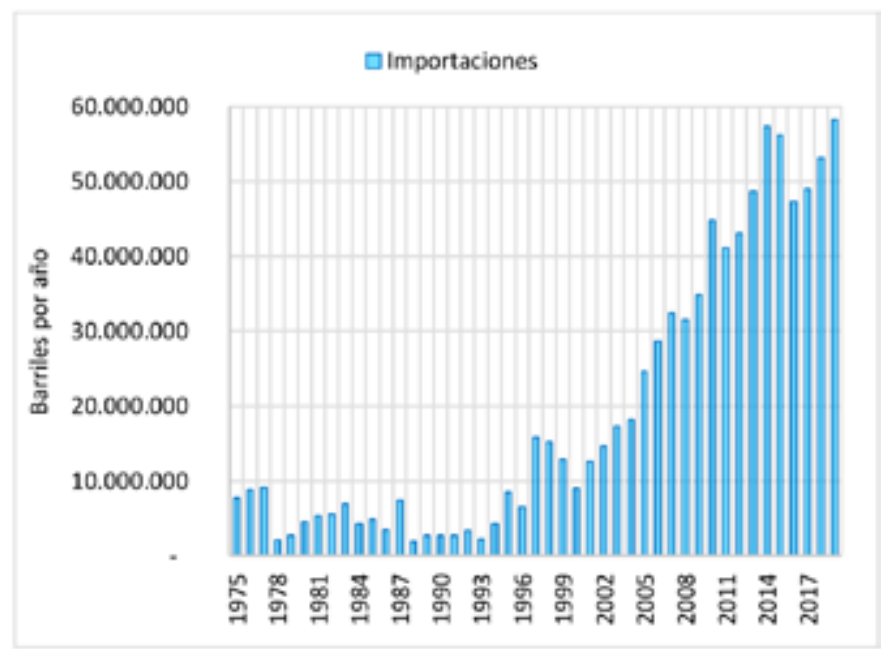

Figura 2. Volumen anual de importaciones de combustibles en el Ecuador. Fuente: EP Petroecuador.

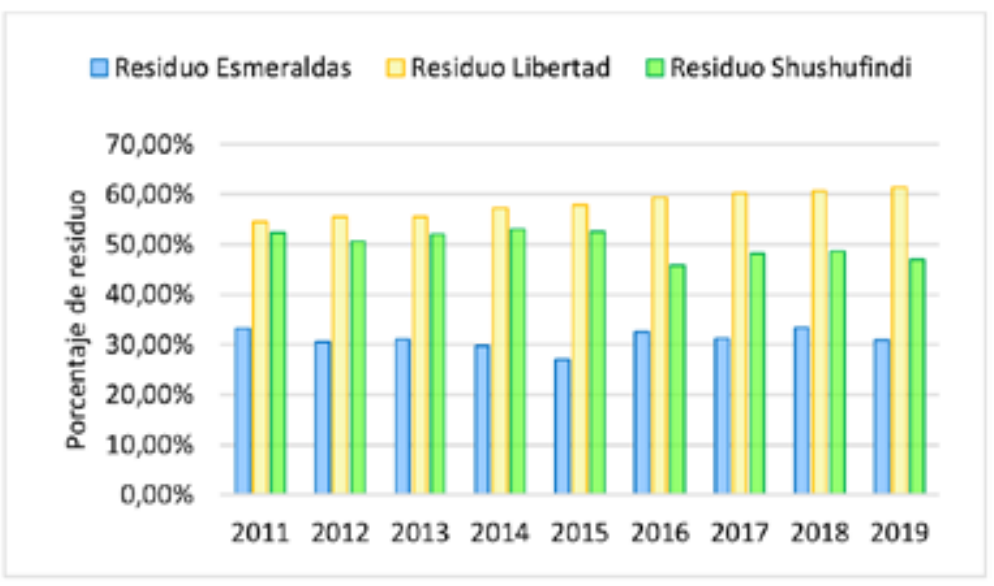

Figura 3. Residuo generado por refinerías.

Fuente: EP Petroecuador. 
expresión: valor económico de las ventas - costo económico de las compras - costos de operación variables. Para el estudio de caso, la expresión matemática (Ec. 1) fue ajustada a la realidad del parque refinador del país, incluyendo los costos de otros productos que se usan para el blending, establecido de la siguiente manera:

Margen neto de refinación

$=$ Ingresos totales - costos por materia prima - Costos por productos para blending - Costos de operación

De lo cual se deriva a lo siguiente:

Margen neto de refinación

$=\left(\Sigma\right.$ Volumen Refinados $_{\mathrm{i}}{ }^{*}$ Precio $\left._{\mathrm{i}}\right)$

$-\left(\Sigma\right.$ Volumen Crudo $_{j}{ }^{\star}$ Costo $\left._{j}\right)$

$-\left(\sum\right.$ Volumen Productos para blending ${ }_{k}{ }^{*}$ Costo $\left._{k}\right)$

- Costos de operación

Ec. (1)

Los ingresos incluyen lo recaudado por comercialización de gasolinas, diésel, GLP y otros refinados que se consumen en su mayoría en el interior del país, además de la exportación del fuel oil que es producto de la mezcla entre el residuo producido y cutter stock importado.

El costo por materia prima, se refiere al crudo Oriente que tiene una gravedad API cercana a 23,5 ${ }^{\circ}$ API con 1,5\% de contenido de azufre (Banco Central del Ecuador, 2009), mientras que los costos de los productos para el blending se refieren a los combustibles importados para las mezclas con productos refinados intermedios, para alcanzar los parámetros mínimos de calidad establecidos en la normativa local (consumo interno) e internacional (exportaciones).

Los costos operativos consideran los gastos corrientes (salarios, servicios y mantenimiento de la infraestructura, gastos en energía, entre otros) incurridos por cada refinería. Para este caso se consideró el valor unitario promedio de USD 6,2 dólares por barril, reportado por la gerencia de Refinación de EP Petroecuador.

El éxito para calcular el margen no solo contempla el manejo de datos volumétricos sino también estará dado por el acceso y conocimiento de los precios del petróleo y de los productos refinados en los mercados en donde tienen acceso los flujos de las refinerías. Los precios de los commodities se consideran volátiles y están fuera del control de cualquier participante en las transacciones de compra y venta de hidrocarburos.

Para garantizar el detalle de la información, res- pecto a los valores volumétricos y monetarios se estableció el siguiente diagrama de flujo (ver Figura 4).

Se recabó información tomando en cuenta las principales variables inmersas en la Ec. 1 para cada refinería del país: 1) Carga de petróleo: corresponde a crudo Oriente que es transportado por el sote desde los campos petroleros de la Amazonía ecuatoriana hasta la provincia de Esmeraldas en el terminal de Balao, para ser luego distribuido a la refinería de Esmeraldas. Para abastecer de petróleo a la refinería La Libertad se incurre en un proceso logístico mayor, puesto que es necesario el transporte del petróleo vía cabotaje (buques) desde Esmeraldas a la Libertad. Para la refinería de Shushufindi por su cercanía al área de extracción de petróleo, la entrega se lo realiza directamente de los campos a través de líneas de oleoducto secundarias. La carga a las refinerías se obtuvo de los reportes estadísticos mensuales de EP Petroecuador y el costo por materia prima fue tomado de la base del Banco Central, cuyo valor refleja el precio de las exportaciones de crudo Oriente referido al marcador WTi (ver Figura 5).

2) Productos importados: Se tomó en cuenta los productos que intervienen en los procesos de blending para la obtención de productos finales. Se importa a) cutter stock, para la obtención de fuel oil de exportación ( 0,3 cutter stock $+0,7$ residuo) b) nafta de alto octano NAO, para la obtención de gasolinas $(0,4 \mathrm{NAO}+0,6$ nafta base de las refinerías).

La composición de las mezclas en las refinerías es dinámica y dependerán de las condiciones de la carga y de la operación. Para este estudio los volúmenes fueron estimados de los reportes mensuales de la EP Petroecuador, al igual que su costo. Se consideró el precio de las importaciones cif que realiza el Estado, por lo cual este valor incluirá los costos incurridos en el transporte.

3) Productos refinados finales generados en cada refinería destinados al mercado interno como: gasolinas, diésel, GLP, gasolina de aviación, asfaltos azufre, solventes, fuel oil y residuo y al mercado externo como: fuel oil n. ${ }^{\circ} 4$ y n. ${ }^{\circ} 6$ y en ocasiones gasóleo vGO. Los datos fueron recabados de las bases estadísticas de ARCH y eP Petroecuador.

Los precios de comercialización se estimaron suponiendo un mercado internacional sin considerar rubros de subsidios de comercialización en el mercado local. Se tomó en cuenta los valores de los combustibles disponibles en las bases estadísticas de la Agencia Internacional de Energía comercializados en el golfo de los Estados Unidos y acorde a la calidad de los productos refinados en el Ecuador. Para los precios de los refinados que no se disponía de in- 


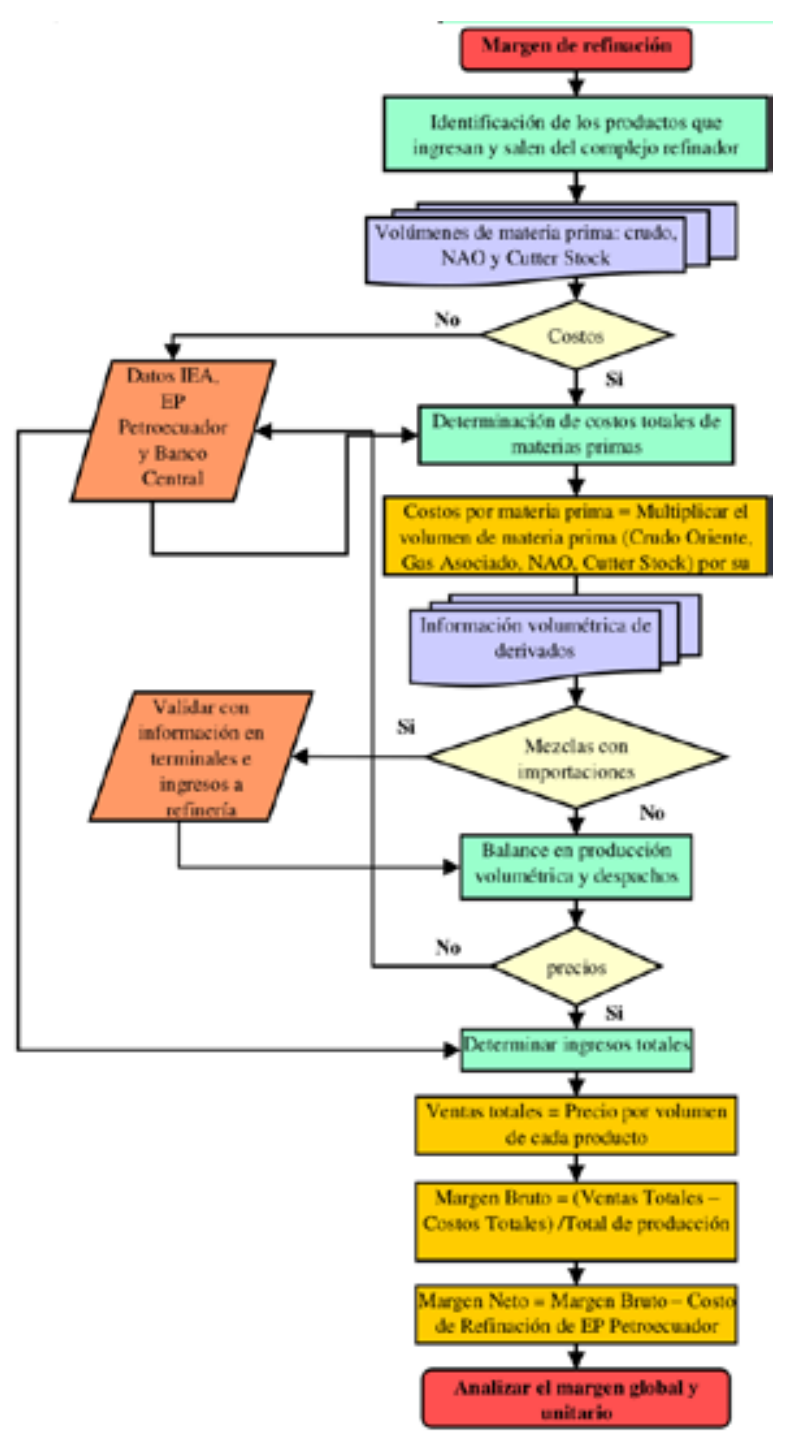

Figura 4. Diagrama de flujo para determinar el margen de refinación en una refinería del Ecuador.

formación (por diferenciación de calidad), se estimó un precio con referencia al combustible de mayor calidad.

\section{Explorando el margen de refinación en otras refinerías}

La Organización de Países Exportadores de Petróleo (OPEP), la Agencia Internacional de Energía (IEA) у вр son algunas de las agencias que realizan análisis mensuales de los márgenes de refinación de las principales regiones del mundo considerando el tipo de crudo y la configuración de cada refinería. Los principales centros de refinación analizados corresponden a infraestructuras de alta y profunda conversión. Por ejemplo, en las refinerías ubicadas en la región del golfo de los Estados Unidos se emplea en su mayoría crudo wTI, para las regiones de Europa y Rusia se consume crudo Brent y

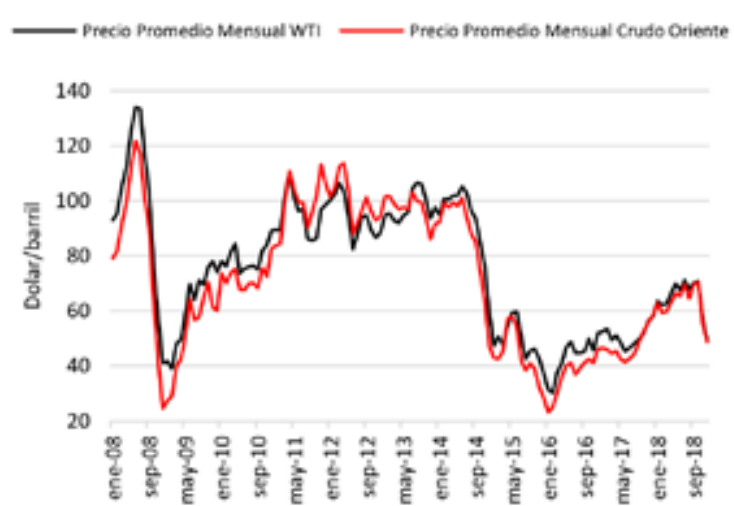

Figura 5. Histórico de precio del crudo WTI y crudo Oriente. Fuente: Banco Central del Ecuador (2019).

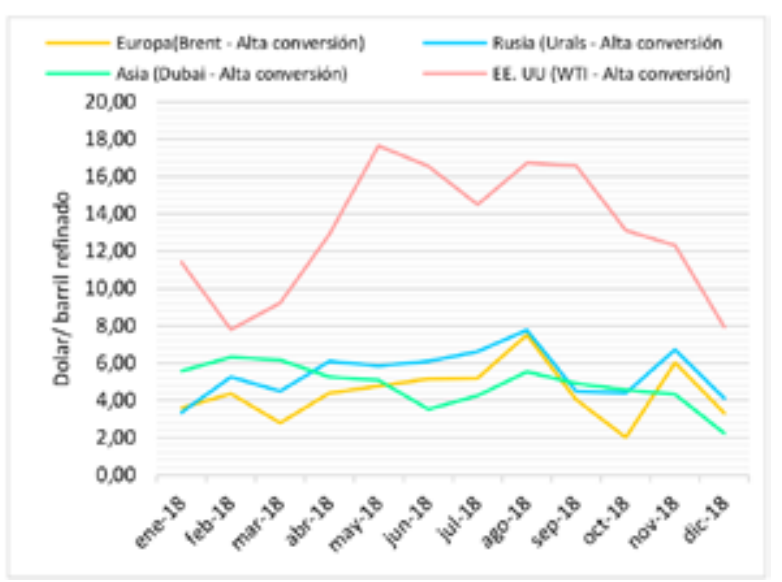

Figura 6. Comparación del margen de refinación por regiones. Fuente: IEA, Oil market report (2019).

Urals, respectivamente, mientras que para Asia el consumo del crudo corresponde al Dubái.

Para contrastar los resultados del presente estudio se tomó en cuenta el benchmark proporcionado por la Agencia Internacional de Energía, que muestra el comportamiento del negocio de refinación de manera mensual y diferencia el margen bruto obtenido por la calidad del crudo procesado en refinerías de alta conversión. En el 2018 se observa que los márgenes de refinación regionales cayeron en varios meses como indica la figura 6 , debido a que los productos derivados de petróleo incrementaron su almacenamiento por una desaceleración estacional de la demanda (ver Figura 6). La caída del margen en la región de Estados Unidos fue una de las más significativas en comparación a otras regiones (International Energy Agency, 2019). 
El análisis regional nos permite comparar el rendimiento del parque refinador del Ecuador con otros complejos refinadores para medir su competitividad en los mercados internacionales, considerando variables como calidad de la materia prima, complejidad de las refinerías y la eficiencia en las operaciones.

\section{DISCUSIÓN DE RESULTADOS}

En el periodo analizado, el parque refinador procesó en promedio 160 mil barriles por día, reflejando un factor de operación del $91 \%$ con respecto a su capacidad nominal. La figura 7 indica la capacidad de operación en función de la carga de petróleo que procesan mensualmente las refinerías y que depende de la planificación operativa (mantenimientos) y de las paradas no programadas (cortes de energía, fallas operativas y eventos emergentes) (ver Figura 7).

Como resultado del proceso de refinación se muestra la producción de los principales productos derivados a nivel nacional. En 2018 se produjeron 2,3 millones de barriles de GLP, 13,8 millones de barriles de gasolinas, 12,6 millones de barriles de diésel y 41 millones de barriles de otros derivados que incluyeron solventes, asfaltos, azufre, fuel oíl, naftas base y crudo residual (ver Figura 8).

Los precios promedio mensuales del crudo Oriente y de los principales refinados muestran una tendencia paralela con respecto al crudo marcador WTI, estos precios corresponden a los productos identificados en la oferta nacional que generan las refinerías, de acuerdo con las bases estadísticas publicadas por la Agencia Internacional de Energía, representado en la figura 9 (ver Figura 9).

A continuación, se muestra la tabulación de los flujos mensuales en volumen y usD que entran y salen del proceso de refinación nacional, previa a determinar los valores del margen bruto (ver Tabla 1).

Tabla 1. Entrada y salida de productos en el parque refinador del Ecuador

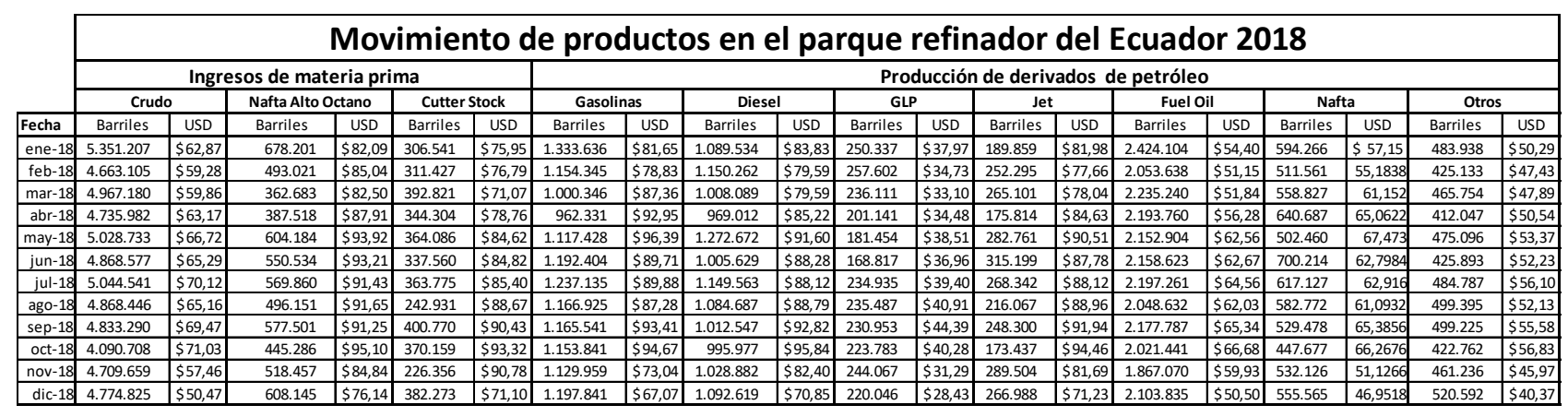

Fuente: EP Petroecuador 2018, Banco Central e IEA.

La tabla 2 muestra la tabulación de los valores en usD de los ingresos obtenidos por la producción de derivados, costos incurridos por materia prima (petróleo y productos importados) y el margen mensual (ver Tabla 2).

Tabla 2. Cálculos para determinar el margen de refinación

\begin{tabular}{|r|cc|c|c|c|}
\cline { 2 - 6 } \multicolumn{1}{c|}{} & \multicolumn{5}{c|}{ Margen de refinación del parque refinador del } \\
Ecuador \\
\hline Mes & $\begin{array}{c}\text { Costos por } \\
\text { materia prima }\end{array}$ & $\begin{array}{c}\text { Ventas de } \\
\text { derivados }\end{array}$ & $\begin{array}{c}\text { Margen } \\
\text { global }\end{array}$ & $\begin{array}{c}\text { Margen bruto } \\
\text { unitario }\end{array}$ \\
\hline ene-18 & $\$ 415.376 .809$ & $\$ 415.471 .890$ & $\$ 95.081$ & $\$ 0,01$ \\
\hline feb-18 & $\$$ & 342.279 .406 & $\$ 364.526 .911$ & $\$ 22.247 .504$ & $\$ 3,83$ \\
\hline mar-18 & $\$$ & 355.170 .110 & $\$ 368.477 .451$ & $\$ 13.307 .342$ & $\$ 2,30$ \\
\hline abr-18 & $\$$ & 360.374 .581 & $\$ 379.810 .706$ & $\$ 19.436 .125$ & $\$ 3,49$ \\
\hline may-18 & $\$ 4$ & 423.057 .699 & $\$ 450.815 .286$ & $\$ 27.757 .587$ & $\$ 4,63$ \\
\hline jun-18 & $\$$ & 397.820 .904 & $\$ 431.160 .407$ & $\$ 33.339 .503$ & $\$ 5,58$ \\
\hline jul-18 & $\$$ & 436.894 .606 & $\$ 453.266 .372$ & $\$ 16.371 .766$ & $\$ 2,64$ \\
\hline ago-18 & $\$ 384.238 .554$ & $\$ 415.717 .797$ & $\$ 31.479 .242$ & $\$ 5,39$ \\
\hline sep-18 & $\$$ & 424.732 .623 & $\$ 440.600 .211$ & $\$ 15.867 .588$ & $\$ 2,70$ \\
\hline oct-18 & $\$$ & 367.463 .485 & $\$ 418.566 .191$ & $\$ 51.102 .707$ & $\$ 9,39$ \\
\hline nov-18 & $\$ 335.172 .878$ & $\$ 358.903 .412$ & $\$ 23.730 .534$ & $\$ 4,27$ \\
\hline dic-18 & $\$ 3$ & 314.450 .107 & $\$ 336.381 .342$ & $\$ 21.931 .235$ & $\$ 3,68$ \\
\hline
\end{tabular}




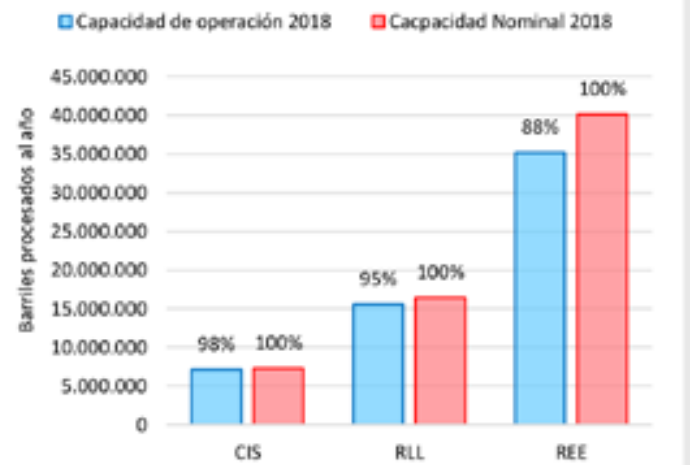

Figura 7. Porcentaje de operación del parque refinador en función de la carga procesada. Fuente: EP Petroecuador (2018).

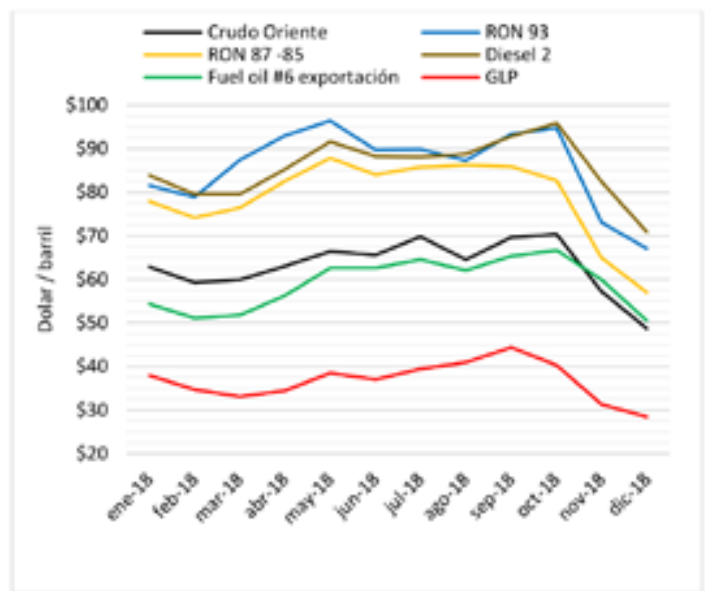

Figura 9. Precios internacionales de productos derivados de petróleo. Fuente: Agencia Internacional de Energía.

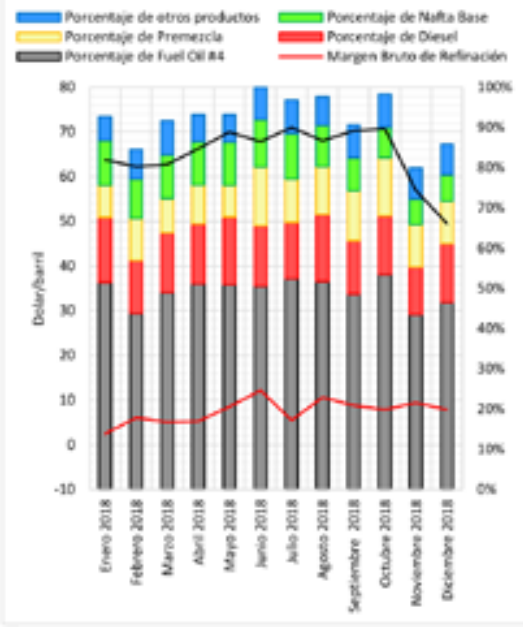

Figura 11. Margen bruto de refinación 2018, refinería La Libertad.

\section{MARGEN DE REFINACIÓN POR REFINERÍA}

Para cada centro de refinación se levantó la información necesaria y se analizó su comportamiento en el 2018, siguiendo la misma metodología aplicada en el análisis nacional. En las figuras 10, 11 y 12 se muestran la composición y dinámica de la producción de derivados de petróleo (barras en colores), el margen bruto unitario y el precio de referencia del petróleo WTI (línea continua) de manera mensual.

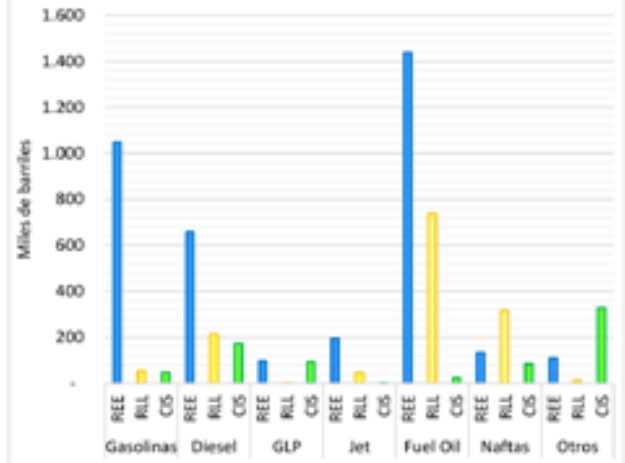

Figura 8. Producción nacional de derivados por refinería. Fuente: EP Petroecuador (2018).

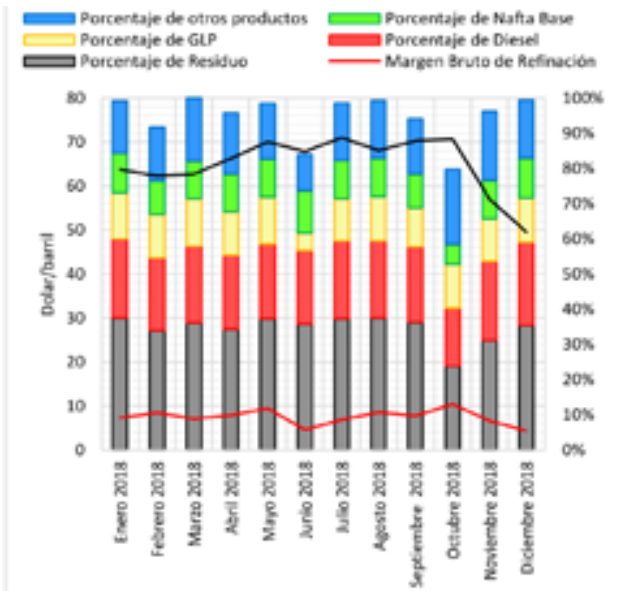

Figura 10. Margen bruto de refinación 2018. Complejo industrial Shushufindi.

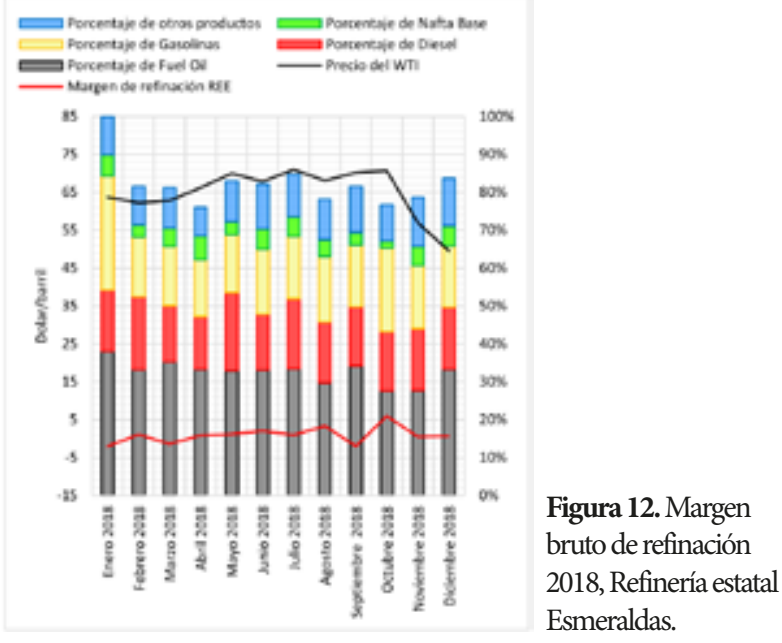

En las tres refinerías se muestra que la dinámica del margen bruto es proporcional a las fluctuaciones del precio del petróleo WTI, además, es evidente las afectaciones que sufre este indicador por mermas en la eficiencia de la operación. De manera específica para el Complejo Industrial Shushufindi (ver Figura 10) el margen bruto promedio anual fue de USD 7,44 por barril producido y se muestra su afectación por la variación en la composición de la producción de 
derivados y disminución del factor de planta de la refinería (disminución en la producción de GLP por mantenimiento programado de la planta de gas) en junio del 2018.

Para la refinería La Libertad (ver Figura 11) el margen bruto de refinación fue de aproximadamente UsD 8,50 por barril producido. Se muestra que en el mes de julio el margen tuvo una disminución por mayor producción de residuo, mientras que en noviembre, pese a que el WTI experimentó una disminución, el margen se mantuvo estable por la disminución de la producción de residuo y la estabilidad de los productos livianos con mayor precio en el mercado.

Para la refinería estatal Esmeraldas (ver Figura 12) el margen de refinación obtenido fluctuó entre USD -2 a 6 dólares por barril producido, lo cual es contradictorio si lo comparamos con el margen obtenido en las refinerías de menor complejidad como La Libertad y Shushufindi.

De acuerdo con los autores, la falta de transparencia y detalle en la información estadística disponible sobre los volúmenes de las importaciones para las mezclas en los procesos intermedios de refinación, puede ser el punto de inflexión para la obtención de valores erróneos, por lo cual se recomienda que, para que este estudio sirva como información sobre la política energética, se involucre a los actores del ciclo de la política para corroborar los datos en trabajos futuros.

Finalmente, en la figura 13 se muestra un comparativo de los resultados entre refinerías nacionales frente a las refinerías de alta y profunda conversión del golfo de los Estados Unidos. Como se puede observar, la dinámica del comportamiento del margen es similar, sin embargo, es clara la pérdida de competitividad de las refinerías nacionales de baja y media conversión con refinerías de mayor volumen y complejidad superior.

\section{CONCLUSIONES}

- Los resultados a priori de este estudio no pretenden informar la política energética, sino más bien mostrar y transparentar el proceso metodológico para el análisis del desempeño de una refinería con el fin de proporcionar métricas de evaluación, las mismas que deben ser utilizadas y validadas por los diferentes actores en el ciclo de la política energética. - Este estudio presenta una metodología para la evaluación del desempeño de las refinerías en el Ecuador, considerando no solo la materia prima utilizada y la producción de derivados, sino también considera todos los flujos intermedios (blending) que entran y salen de los procesos. De acuerdo a los resultados obtenidos las refinerías nacionales mantuvieron un margen bruto en el 2018 de entre USD 2,30 y 9,40 dólares por barril procesado, frente a USD 8 y 17 dólares obtenido por refinerías con mayor complejidad en otras regiones.

- La intensidad de los procesos intermedios de mezclado por falta de modernización tecnológica de las refinerías nacionales encarece la producción de los derivados y las vuelve menos competitivas si las comparamos con otras refinerías a nivel mundial. En el 2018 se importaron aproximadamente 16 millones de barriles de naftas de alto octanaje y 4 millones de barriles de cutter stock para la obtención de gasolinas y fuel oil para su comercialización.

- Analizando el margen neto de las refinerías que incluye el costo de operación, el panorama refleja que, para llegar al punto de equilibrio económico de las refinerías, el margen bruto deberá estar por encima de USD 6,24 dólares por barril procesado, que representa el costo de operación promedio por barril procesado, de acuerdo a la información de EP Petroecuador.

- El margen bruto promedio mensual para el periodo de análisis en el complejo industrial Shushufindi fue de USD 7,44, en la refinería La Libertad de USD 8,48 y en la refinería estatal Esmeraldas de USD 1,24, lo cual, como se mencionó en la sección correspondiente del estudio, no es coherente con la complejidad de las refinerías, pues a mayor complejidad y mayor volumen se esperaría que el margen bruto mejore frente a refinerías de menor complejidad y de menor capacidad instalada.

- Finalmente, se recomienda que para futuros trabajos en el tema de refinación, los datos de las estadísticas nacionales sean revisados y validados con información primaria para que estos trabajos de investigación sirvan como insumos para informar sobre la política energética del Ecuador. 


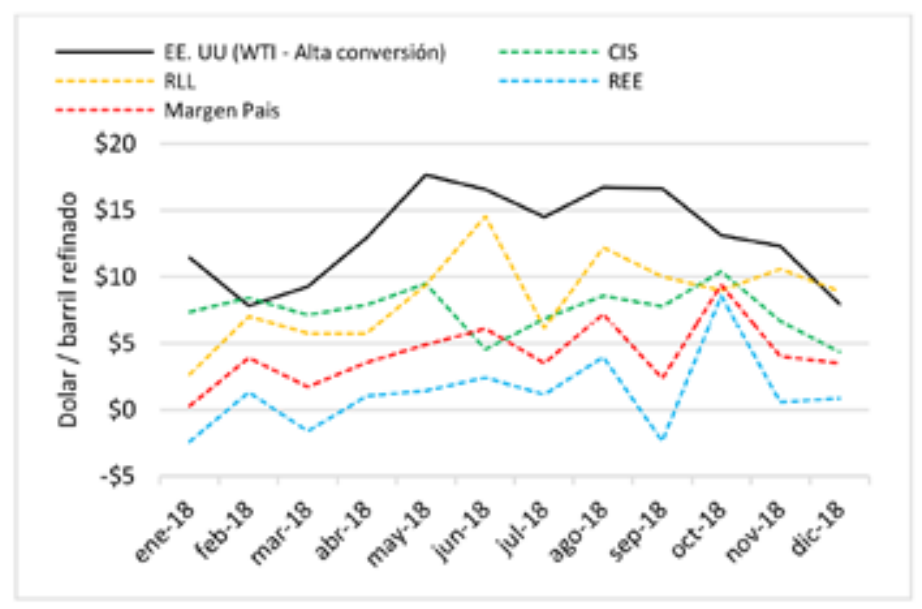

Figura 13. Comparación del margen nacional con refinerías de Estados Unidos.

\section{REFERENCIAS}

Baquero, P. 2006. Las ganancias y pérdidas. En G. Fontaine (ed.), Petróleo y desarrollo. Quito: Sede Flacso, p. 149.

BCE. 2009. Precios de los crudos ecuatorianos, s. l.: Banco Central del Ecuador .

BCE. 2018. Reporte del Sector Petrolero, s. 1.: s. n.

BP. 2020. Regional refining margins. Statistical Review of World Energy, p. 27.

Carrillo, C. 2020. Análisis del margen de refinación en el sector hidrocarburifero del Ecuador, utilizando la información en tiempo real de la Agencia de Regulación y Control Hidrocarburífero en el periodo 2018-2019. [En línea] Disponible en: http://www.dspace.uce.edu.ec/ handle/25000/21558

Cuesta, S. 2019. Gobierno anuncia concesión de refinería y construcción de una nueva en Esmeraldas [Entrevista] (15 agosto 2019).

DEN 1. 2020. La gestión conjunta entre la empresa privada y la EP Petroecuador para la operación de la Refinería de Esmeraldas, s.l.: s.n.

El-Gendy, N. S. and Speight, J. G. 2015. Refinery configuration. En T. \&. F. Group (ed.), Handbook of refinery desulfurization. s.l.: CRC Press, pp. 83-86.

EP Petroecuador. 2018. Gerente general de EP Petroecuador supervisó operaciones de exportación de crudo desde el Terminal Marítimo Balao. [En línea] Disponible en: https://www.eppetroecuador.ec/?p=8228

EP Petroecuador 2018. Informe Estadístico 1972-2017 (45 años). Quito: EP Petroecuador.

EP Petroecuador 2015. Análisis estadístico anual de Petroecuador.

EP Petroecuador 2019. Análisis estadístico anual, s. 1.: Gerencia de Refinación.

Gary, J. H., Handwerk, G. E. and Kaiser, M. J. 2004. Refining margins. En T. \&. F. Group (ed.) Petroleum refining tecnology and economics. s. 1.: CRC Press, p. 22.

Hirshfeld, D. 2015. Stillwater Associates. [En línea] Disponible en: https://stillwaterassociates.com/crack-spread-a-quick-and-dirty-indicator-of-refining-profitability [Último acceso: 24 junio 2020].

International Energy Agency. 2019. Margins. Oil market report, p. 25.

International Energy Agency. 2020. Margins. Oil market report.

Lluch Urpi, J. 2011. Tecnología y margen de refino del petróleo. Díaz de Santos s. A. (ed.). Madrid: Instituto Superior de la Energía.

opec. 2011. Brent, West Texas, Arab Light... Conoza al detalle qué representa cada tipo de petróleo, cuándo, cómo $y$ dónde se negocia. [En línea] Disponible en: https:// www.expansion.com/especiales/petroleo/crudos.html

OPEC. 2019. Product markets and refinery operations. Monthly oil market report.

Ortiz, R. 2020. El Gobierno nacional presentó la licitación para construir una refinería de alta conversión en la Costa ecuatoriana [Entrevista] (11 marzo 2020).

Petroecuador E. P. 2019. Informe estadístico anual 1972-2019, Quito: Subgerencia de Planificación y Control de Gestión.

Población, J. and Serna, G. 2016. Is the refining margin stationary? International Review of Economics and Finance, p. 170.

Ramkumar, M. 2016. marketrealist.com. [En línea] Disponible en: https://marketrealist.com/2016/11/refining-margins-key-indicators-refining-profitability/ [Último acceso: 12 julio 2020].

RPI. (2016). Plan Institucional 2016-2019, s. 1.: Gerencia de Planificación y Procesos. 